Further work is required to establish its suitability for detecting the other organisms claimed.

\section{P170 RISK FACTORS FOR MYCOPLASMA GENITALIUM INFECTION IN SYMPTOMATIC MALES, FEMALES AND MEN WHO HAVE SEX WITH MEN FROM THREE CLINICAL SETTINGS IN LONDON}

${ }^{1}$ Claire Broad ${ }^{*},{ }^{2,1}$ Emma Harding-Esch, ${ }^{1}$ Mark Harrison, ${ }^{1}$ Clare Soares, ${ }^{1}$ Sebastian Fuller ${ }^{2}$ Sandra Okala, ${ }^{3,2}$ John Saunders, ${ }^{4}$ Tristan Barber, ${ }^{5}$ Phillip Hay, ${ }^{1,2}$ Tariq Sadiq. ${ }^{1}$ St George's University of London, London, UK; ${ }^{2}$ Public Health England, London, UK; ${ }^{3}$ Central and Northwest London NHS Foundation Trust, London, UK; ${ }^{4}$ Chelsea and Westminster Hospital NHS Foundation Trust, London, UK; ${ }^{5}$ St George's University Hospitals NHS Foundation Trust, London, UK

\subsection{6/sextrans-2016-052718.220}

Background/introduction Mycoplasma genitalium (MG), a sexually transmitted infection (STI), is increasingly recognised as a cause of major reproductive health sequelae. Treatment has become increasingly difficult due to macrolide and fluoroquinolone antibiotic resistance. MG is not routinely tested for in most UK genitourinary medicine (GUM) clinics, and limited risk-factor data exist for infection in at-risk populations and in different anatomical sites.

Aim(s)/objectives To determine risk factors for MG infection in symptomatic male and female patients accessing three London GUM clinics.

Methods Patients aged $\geq 16$ years, symptomatic of an STI (or Chlamydia, Gonorrhoea, Trichomonas vaginalis, or non-specific urethritis contact) were consented. Additional-to-routine samples provided were vulvovaginal swab (VVS) (females), first void urine (FVU) (men-who-have-sex-with-women (MSW), (menwho-have-sex-with-men (MSM)), pharyngeal and rectal swabs (MSM). Samples were tested using the FTD Urethritis Plus Test kit and positives confirmed by Polymerase Chain Reaction. Risk factors were analysed using univariate and multivariate logistic regression.

Results MG was detected in: 10.7\% (95\% CI 7.9\%-13.5\%) patients; 7.9\% (95\% CI 4.86\%-10.94\%) VVS; 19.4\% (95\% CI 11.76\%-27.04\%) MSW urine; 1.6\% (95\% CI 0\%-4.72\%) MSM urine; 0\% MSM pharynx; $8.1 \%$ (95\% CI 1.31\%-14.89\%) MSM rectum.

Abstract P170 Table 1 Risk factors associated with MG infection

\begin{tabular}{llll}
\hline Risk & Male & $\begin{array}{l}\text { Odds Ratios (95\% } \\
\text { Confidence interval) } \\
\text { Univariate MSW }\end{array}$ & $\begin{array}{l}\text { Odds Ratios (95\% } \\
\text { Confidence interval) } \\
\text { Univariate Females }\end{array}$ \\
\hline Age & $16-19$ & $1^{*}$ & $1^{*}$ \\
& $20-24$ & $0.06(0.01-0.61)$ & $0.46(0.15-1.40)$ \\
& $25-34$ & $0.16(0.02-1.08)$ & $0.26(0.08-0.79)$ \\
Ethnicity & $34-44$ & $0.24(0.03-2.03)$ & $0.08(0.01-0.68)$ \\
& White & 1 & 1 \\
& Mixed & $10.00(0.61-162.66)$ & $2.98(0.88-10.13)$ \\
& Asian & $7.00(0.46-96.44)$ & $2.13(0.24-18.76)$ \\
Symptoms & Black & $8.33(1.78-38.97)$ & $1.58(0.60-4.19)$ \\
& Discharge & 1 & - \\
Gonorrhoea Contact & Pain & $0.68(0.24-1.89)$ & - \\
& No & - & - \\
& Yes & - & $11.5(1.54-85.64)$ \\
\hline
\end{tabular}

*Only ages 16-19 remained a risk factor in the multivariate analysis
Discussion/conclusion MG positivity was highest in MSW compared to the other patient groups, with younger age being the only risk factor for infection, remaining after multivariate analysis. The presence of rectal MG despite a lack of urogenital infection in MSMs warrants further investigation with a larger cohort. Overall the results indicate high MG positivity across symptomatic male and female populations.

\section{P171 RAPID RELIABLE HIV POINT OF CARE TESTING}

Clare Wood*, Susanna Currie, Louise Hesketh, Andrew Turner, Debbie Thomas, Helen Holt, Orla McQuillan. Manchester Royal Infirmary, Manchester, UK

\subsection{6/sextrans-2016-052718.221}

Background Our outreach HIV Point of care testing (POCT) programme changed from $4^{\text {th }}$ generation testing to $3^{\text {rd }}$ generation POCT kits in August 2014, which led to a significantly quicker turnaround time for results and greater convenience for both outreach staff and patients. We continued to confirm all POCT serology by conventional laboratory testing.

Aims To compare $3^{\text {rd }}$ and $4^{\text {th }}$ generation POCT in clinical practice and review the need for laboratory confirmation of all samples.

Methods The INSTI ${ }^{\mathrm{TM}}$ HIV-1/HIV-2 Antibody Test was used for POCT testing at a city centre outreach service from August 2014 until July 2015. All samples were also tested in parallel, in realtime, by standard laboratory tests for HIV. Results were compared retrospectively.

Results POCT was provided for 399 patients. 31 patients were excluded. Of the remaining 368 patients, there were 6 true positive results $(1.6 \%)$ and no false-negatives or false-positives. By contrast, our previous evaluation of Alere Determine ${ }^{\mathrm{TM}} 4^{\text {th }}$ generation testing, with a sample size of 367 , found 3 true positives $(0.8 \%) ; 2$ false positives $(0.6 \%)$; and 3 false negatives $(0.8 \%)$, leading to negative predictive value $99.2 \%$; positive predictive value $60 \%$; sensitivity $50 \%$; specificity $99.4 \%$. This was a significant underperformance in clinical practice compared with advertised values.

Discussion INSTI ${ }^{\mathrm{TM}}$ is outperforming Alere Determine ${ }^{\mathrm{TM}}$ in our local experience. We intend to continue using $3^{\text {rd }}$ generation POCT in our outreach programme. Given INSTI ${ }^{\mathrm{TM}}$, $s$ performance, the question now raised is can we consider moving away from carrying out backup serology in all cases?

\section{P172 HIGH HIV INCIDENCE IN MSM DIAGNOSED WITH EARLY SYPHILIS: A ROLE FOR PREP?}

Nicolo Girometti, Angela Gutierrez, Nneka Nwokolo, Alan McOwan, Tara Suchak* Gary Whitlock. ${ }^{56}$ Dean Street, Chelsea \& Westminster Hospital, London, UK

\subsection{6/sextrans-2016-052718.222}

Background. Understanding the risk factors for HIV acquisition allows targeted interventions to reduce HIV transmission such as PrEP.

Aims/Objectives. To evaluate HIV incidence in HIV-negative MSM with early syphilis infection.

Methods. A retrospective case-note review of MSM who were diagnosed with early syphilis between January and June 2014 at a London sexual health clinic.

Results. 206 MSM were diagnosed with early syphilis: $110 \mathrm{HIV}$ negative; 96 HIV-positive. For 110 HIV-negative MSM, median 